\title{
What dominates the X-ray emission of normal galaxies?
}

\author{
Ann E. Hornschemeier ${ }^{1}$, Anna Wolter ${ }^{2}$ and Dong-Woo Kim ${ }^{3}$ \\ ${ }^{1}$ NASA Goddard Space Flight Center, Laboratory for X-ray Astrophysics, \\ Code 662, Greenbelt, MD 20771, \\ email: Ann.Hornschemeier@nasa.gov \\ ${ }^{2}$ INAF-Osservatorio Astronomico di Brera, \\ Via Brera 28, 20121, Milano, Italy \\ email: anna.wolter@brera.inaf.it \\ ${ }^{3}$ Smithsonian Astrophysical Observatory \\ 60 Garden Street, Cambridge, MA 02138 \\ email: kim@cfa.harvard.edu
}

\begin{abstract}
X-ray surveys of normal galaxies, i.e. those that do not host actively supermassive black holes, have revealed important information on the nature of accreting stellar-mass compact objects (neutron stars and black holes), constraints on populations of possible intermediate-mass black holes $\left(10^{2-5} M_{\odot}\right)$, and on the reservoir of materials in the hot interstellar medium of the most massive galaxies. Here we summarize briefly the results of Chandra and NuSTAR surveys of several samples of galaxies covered during the 2015 IAU General Assembly. This includes a comprehensive study of six nearby starburst galaxies by the NuSTAR mission, of high-redshift galaxies from the $6 \mathrm{Ms}$ Chandra Deep Field South for which evolutionary trends in X-ray emission over cosmic time have been measured, of collisional ring galaxies which are excellent local environments for studying intermediate-mass black holes and of elliptical galaxies which are ideal for study of the hot gas reservoirs containing the effects of stellar and AGN feedback.
\end{abstract}

Keywords. galaxies: starburst, galaxies: high-redshift, X-rays: galaxies, X-rays: binaries, black hole physics, X-rays: ISM, galaxies: elliptical and lenticular, cD, (cosmology:) early universe

\section{Introduction}

The X-ray emission from "normal" galaxies, that do not contain actively accreting supermassive black holes, arises from many sources. The overall flux is heavily dominated by accreting neutron star and black hole binary systems, possible intermediate-mass black hole systems, and the hot interstellar medium. The X-ray output from normal galaxies can rival that of $\mathrm{AGN}$ at the critical epoch of reionization $(6 \lesssim z \lesssim 10)$ and may have a critical role in the heating of the Intergalactic Medium (IGM) at early times in the Universe (Cosmic Dawn; $10 \lesssim z \lesssim 20$ ) before the IGM was fully ionized (Fragos et al. 2013; Mesinger et al. 2014; Pacucci et al. 2014, Ryu et al. 2016).

We outline results from surveys of galaxies over a range of distances and activity levels, including the deepest X-ray surveys of the distant Universe and surveys of compact objects in spatially-resolved galaxies from $4 \lesssim d \lesssim 50 \mathrm{Mpc}$. These proceedings cover recent results with the highest angular resolution imaging $\mathrm{X}$-ray observatories in orbit across the 0.5-80 keV bandpass: the Chandra X-ray Observatory at $0.5-10 \mathrm{keV}$ and the NuSTAR Explorer mission at $10-80 \mathrm{keV}$.

Directly observable signatures of accretion in X-ray binaries (XRBs) are unique probes of both the formation and evolution of compact objects such as neutron stars (NS) and black holes (BH) and of the binary phase of stellar evolution. The hot interstellar medium 


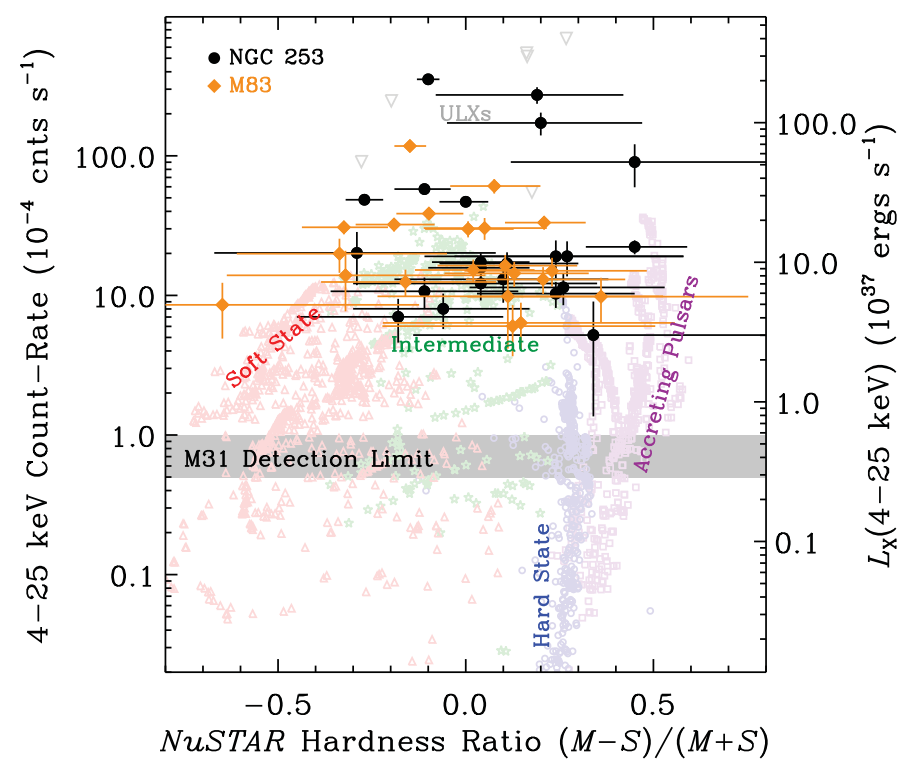

Figure 1. NuSTAR hard X-ray studies of extragalactic XRBs have shown a preponderance of luminous intermediate-state binaries. Shown is $L_{\mathrm{X}}$ versus hardness ratio $(S=4-6 \mathrm{keV}$ and $M=6-12 \mathrm{keV})$ for the two deepest and nearest $\left(N u S T A R t_{\exp } \sim 400-500 \mathrm{ks} ; \mathrm{d} \sim 4 M p c\right)$ starburst galaxies in the NuSTAR-Chandra sample (NGC 253 and M83; Wik et al. 2014; Yukita et al. 2016). The count rates are scaled to $4 \mathrm{Mpc}$, the distance of NGC 253 \& M83. The background colorful points are comparison MW XRBs (Zezas et al. 2016, in prep) and NuSTAR-observed ULXs are shown with grey triangles.

(ISM) of galaxies is the repository of heat and metals from generations of supernovae and in some cases may escape from galaxies to pollute the IGM. Both sources of X-ray emission may play very important roles at early times in our Universe in heating the IGM. Our understanding of accreting binaries and hot gas in galaxies has been revolutionized in the past fifteen years owing to the sub-arcsecond angular resolution of the Chandra X-ray Observatory, enabling spatially-resolved studies of local galaxies and the sensitive measurement of the total X-ray emission from very distant $(z \gtrsim 4)$ galaxies at a time when the Universe was only a tenth of its current age.

\section{Recent X-ray observations of normal galaxies}

Hard X-ray emission from starburst galaxies with NuSTAR(Hornschemeier) .

Star-forming galaxies are the most numerous X-ray emitting extragalactic population in the Universe (e.g., Lehmer et al. 2012), but before NuSTAR we knew next to nothing about their hard (10-30 keV) X-ray emission. Understanding the $0.5-30 \mathrm{keV}$ Spectral Energy Distribution (SED) of star-forming galaxies is important. For example, when we observe galaxies at high redshift $(z \gtrsim 3)$ we observe the rest-frame harder X-ray emission $(E>2 \mathrm{keV})$ even at the softest energies available (the $0.5-8 \mathrm{keV}$ bandpass of Chandra corresponds to $2.0-32 \mathrm{keV}$ at $z \approx 3$ ). As we reach the critical reionization epoch of the Universe $(6<z<10)$, the Chandra bandpass corresponds to $4.0-64 \mathrm{keV}(z=7)$, while the NuSTAR bandpass corresponds to 3.0-80 keV (Harrison et al. 2013). The combination of Chandra and NuSTAR enables simultaneous measurement across 0.5$30 \mathrm{keV}$, ensuring we can accurately extrapolate from the directly-observed harder X-ray bandpass into the less accessible $E<2 \mathrm{keV}$ band at high redshifts. The peak sensitivity 


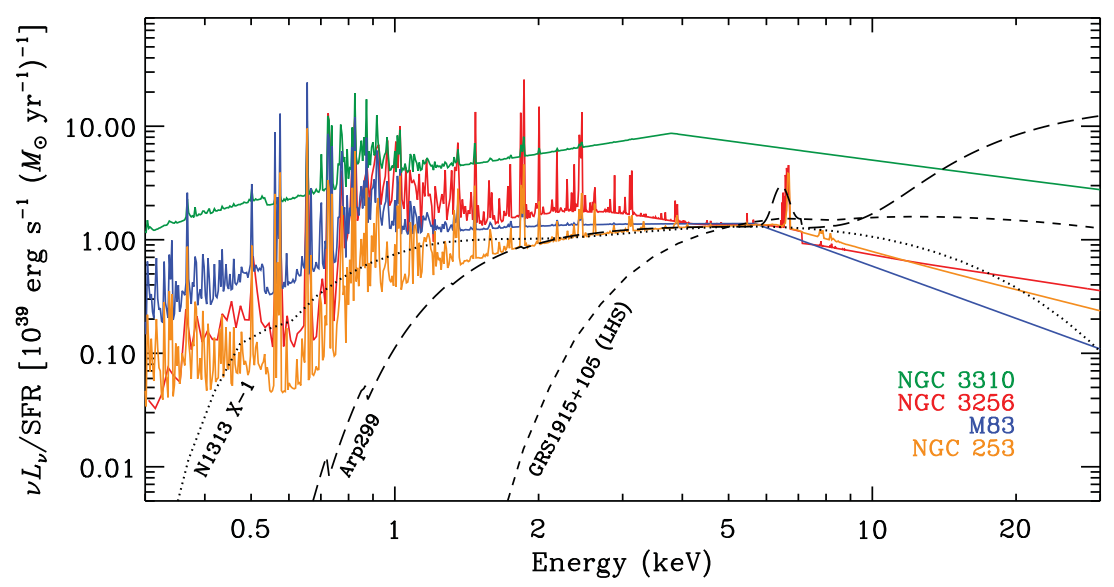

Figure 2. Simultaneous NuSTAR and Chandra observations have permitted the first sensitive constraints on the 0.5-30 keV SED of star-forming galaxies (adapted from Lehmer et al. 2015). Shown are SFR-normalized 0.5-30 keV best-fit SEDs based on nearly simultaneous Chandra/XMM-Newton plus NuSTAR data for four starburst galaxies (Wik et al. 2014; Yukita et al. 2016). The emission lines at $\mathrm{E}<2 \mathrm{keV}$ are due to diffuse gas, not XRBs (e.g., Mineo et al. 2012). Also shown are NuSTAR-constrained SEDs for the ULX NGC 1313 X-1, the luminous BH XRB GRS 1915 in the low/hard state, and the Compton-thick AGN in Arp 299 (an SED with $\Gamma=2$ would be a flat line). All four galaxies show steepening in their SEDs above $\sim 4-6 \mathrm{keV}$, and match best with NGC $1313 \mathrm{X}-1$ which is a BH XRB likely in a super-Eddington accretion mode.

of $N U S T A R$ is in the $10-30 \mathrm{keV}$ bandpass, so hereafter we confine discussion of NuSTAR results to $\mathrm{E}<30 \mathrm{keV}$.

We look to extragalactic starburst environments, as the XRBs that dominate the hard X-ray emission from vigorously star-forming galaxies are often more luminous $\left(L_{\mathrm{X}} \gtrsim 10^{39} \mathrm{erg} \mathrm{s}^{-1}\right)$ than any that can be studied in detail in the Milky Way as there simply are not as many Milky Way XRBs at these higher luminosities.

Due to the highly variable nature of XRBs, simultaneous coverage with $N u S T A R$ and Chandra is needed to constrain the $0.5-30 \mathrm{keV}$ spectrum of XRBs. To this end, a $N u S$ TAR-Chandra survey of six nearby star-forming galaxies (NGC 253, Arp 299, M82, M83, NGC 3256, and NGC 3310) has been conducted. These galaxies were selected to (1) be bright in the 2-7 keV bandpass, and (2) span a moderate range of stellar masses $\left(M_{\star}=1-10 \times 10^{10} M_{\odot}\right)$ and star-formation rates $\left(\mathrm{SFR}=1-80 M_{\odot} \mathrm{yr}^{-1}\right)$.

With the exception of Arp 299 (Ptak et al. 2015 and references therein), these starburst galaxies do not contain actively accreting supermassive black holes and may be studied to determine the overall $0.5-30 \mathrm{keV}$ SED of star-forming galaxies as well as the contribution of XRBs to this overall emission. Hereafter excluding Arp 299, the remaining five starburst galaxies comprise a set of three very nearby ( $\mathrm{d} \sim 4 \mathrm{Mpc}$; M82, NGC 253 and M83) and the two nearest merger systems (d 18 and 39 Mpc; NGC 3310 and NGC 3256). NGC 3256 is also the most X-ray luminous starburst galaxy in the local Universe (Moran et al. 1999).

Chandra-NuSTAR imaging over $0.5-30 \mathrm{keV}$ has allowed sensitive determination of the accretion states of XRBs in star-forming galaxies via their 4-25 keV hardness and luminosities (see Fig. 1; Lehmer et al. 2013; Walton et al. 2013; Wik et al. 2014; Rana et al. 2015; Yukita et al. 2016). Based on these studies, it appears that the bright XRBs, those with $L_{\mathrm{X}} \approx 10^{38-39} \mathrm{erg} \mathrm{s}^{-1}$, in star-forming galaxies are candidate BHs spending the majority of their time in intermediate states as they transition between hard and 
soft states. We caution, however, that although the pulsars are represented well on these diagnostic diagrams, the non-magnetized neutron star binaries (Z-type and atolls) are not shown and are expected to overlap the intermediate-state $\mathrm{BH}$ region of the diagram, particularly at lower X-ray luminosities. With high signal-to-noise NuSTAR spectra NS accretors may be distinguished from BH (Maccarone et al. 2016). Timing studies with $N u S T A R$ have also shown that NS may extend up to very high X-ray luminosities (e.g., the ULX pulsar in M82; Bachetti et al. 2014) so $L_{\mathrm{X}}$ alone is insufficient for separating $\mathrm{BH}$ from NS. Currently, we are exploring distinguishing NS and BH using additional hard X-ray color information (e.g., D. Wik et al. 2016 in prep.)

For starburst galaxies, at $E<2 \mathrm{keV}$, the spectra are known to be dominated by diffuse thermal gas and XRBs. While XRBs likely dominate at $E>10 \mathrm{keV}$, the distribution of XRB accretion states and the overall SED has been less certain until recently. With NuSTAR, we have now measured the $0.5-30 \mathrm{keV}$ SED for a sample of star-forming galaxies for the first time (Wik et al. 2014; Lehmer et al. 2015; Yukita et al. 2016), finding a break at 4-6 keV and a turnover/downward slope above this energy (note colored SEDs in Fig 2). This turnover matches well with e.g., a super-Eddington mode of accretion for stellar-mass BHs (Ultraluminous X-ray, ULX, sources; Gladstone et al. 2009). The $E>$ $10 \mathrm{keV}$ emission scales with SFR, and may exhibit a metallicity dependence (Lehmer et al. 2015). Specifically, the lowest metallicity galaxy, NGC 3310, has the highest overall Xray emission. This is consistent with expectations that in lower metallicity environments stellar winds are weaker, resulting in a larger number of massive black holes (e.g., Linden et al. 2010).

\section{The cosmic evolution of X-ray emission with cosmic time (Hornschemeier).}

The energy output from XRB populations has been modeled via population synthesis from the first galaxies $(z \approx 20)$ until today (Fragos et al. 2013a,b). Interestingly, this work has shown that the X-ray emission from XRBs should dominate over AGN at $z \gtrsim 6$. The long mean-free paths of X-ray photons enable escape from their host galaxies and interaction with the IGM (Fragos et al. 2013a,b; Kaaret 2014). This has major implications for the heating of the IGM and is observable directly via high- $z 21 \mathrm{~cm}$ measurements of the IGM with next-generation radio observatories (e.g., Pacucci et al. 2014). It is the $E<2 \mathrm{keV}$ emission that is most important to early heating and possibly to the reionization of the IGM. Note that due to redshifting, what we observe directly for $z \gtrsim 4$ galaxies is the rest-frame $E \sim 3-30 \mathrm{keV}$ emission; therefore, the $0.5-30 \mathrm{keV}$ SED, measured for local galaxies with Chandra and $N u S T A R$ (see previous section) is a critical input to measuring the X-ray output of high- $z$ galaxies at lower $(E<2 \mathrm{keV})$ energy.

How well constrained is the overall X-ray emission of galaxies at earlier times in the Universe? We can start with our knowledge of local galaxies, where we have observed strong correlations between the $2-10 \mathrm{keV}$ X-ray luminosity and both SFR and stellar mass (e.g., Grimm et al. 2003; Hornschemeier et al. 2005; Lehmer et al. 2010 and references therein). As a reminder, XRB systems are classified by the donor mass as either High Mass X-ray Binaries (HMXBs) or Low Mass X-ray Binaries (LMXBs) and it is expected that HMXB systems will evolve rapidly (within $\sim 10^{7}$ yrs) to an accreting phase after a star formation episode whereas LMXBs should take a much longer time ( $\gtrsim 1$ Gyr) to evolve to an accreting phase and should thus have emission that scales with the total stellar mass of a galaxy. We may write the total $L_{\mathrm{X}}$ from XRBs as $L_{\mathrm{X}}(\mathrm{XRB})=$ $L_{\mathrm{X}}(\mathrm{LMXB})+L_{\mathrm{X}}(\mathrm{HMXB})=\alpha M_{\star}+\beta \mathrm{SFR}$. From this, we may derive expectations for the timescales for the evolution of X-ray emission from galaxies. In the deepest X-ray surveys of the distant Universe, the Chandra Deep Fields, the overall X-ray evolution of galaxies has been shown to agree well with XRB population synthesis models (e.g., 

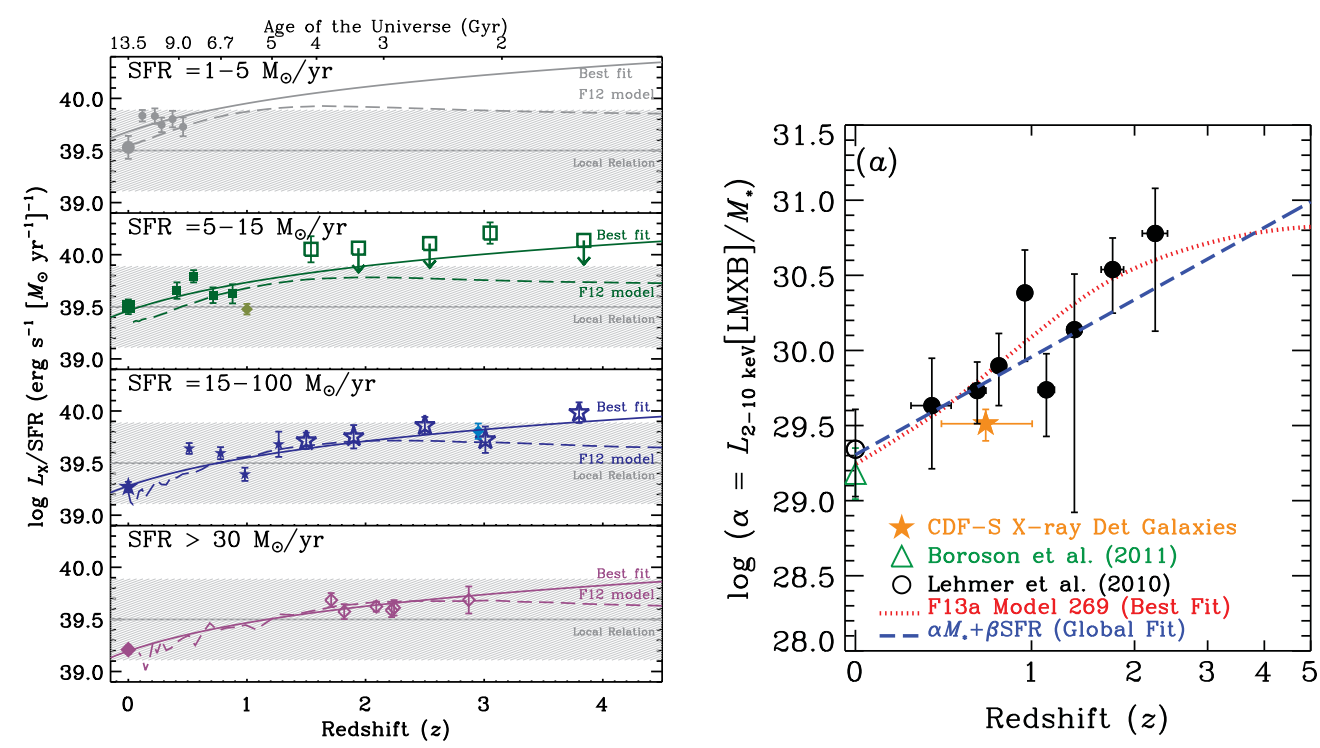

Figure 3. (Left:) Adapted from Basu-Zych et al. (2013a): By studying the X-ray emission from thousands of galaxies in the deepest Chandra surveys, the evolution in the $2-10 \mathrm{keV}$ emission from Lyman Break Galaxies, caused mainly by the metallicity evolution of the Universe, has been demonstrated. (Right:) Using the latest $6 \mathrm{Ms}$ Chandra Deep Field-South data (B. Luo et al. 2016, in prep), the evolution in both $\alpha$ (shown) and $\beta$ (see Lehmer et al. 2016) in the $\mathrm{X}$-ray luminosity from binaries has been measured over cosmic time $\left(L_{\mathrm{X}}(\mathrm{XRB})=\alpha M_{\star}+\beta \mathrm{SFR}\right.$; Lehmer et al. 2016). Using this measured evolution and the evolutionary history of star formation and stellar mass, it is confirmed that the volume X-ray emissivity of galaxies is high at early times in the Universe. The level of overall X-ray emission is significant enough to play a major role in the heating of the primordial IGM at Cosmic Dawn.

Ptak et al. 2001; Hornschemeier et al. 2003; Lehmer et al. 2005; Basu-Zych et al. 2013a). For example, recent work on deep X-ray stacking of thousands of high-redshift $(1 \lesssim z \lesssim 4)$ galaxies using the $4 \mathrm{Ms}$ Chandra Deep Field-South (CDF-S) data, provided the first clear observational evidence of the somewhat subtle evolution of $L_{\mathrm{X}}$ per unit SFR over cosmic time (Basu-Zych et al. 2013a; see Figure 3). This evolution may be attributed to the metallicity evolution of the Universe, and similar to the overall higher X-ray emission mentioned for the low-metallicity galaxy NGC 3310 in the previous section is due to the expected larger population of massive black holes due to weaker stellar winds (e.g., Linden et al. 2010). This picture is further supported by recent observations of localUniverse Lyman Break Analogs which have low metallicity and large numbers of ULX sources (Basu-Zych et al. 2013b, 2016).

Recently, the Chandra Deep Fields have taken an additional step deeper to 6 Ms depth (ultimately this will be $7 \mathrm{Ms}$ depth; B. Luo et al., 2016, in prep.). This new work has allowed a constraint of the evolution of both $\alpha$ and $\beta$ parameters with redshift to $z \lesssim 4$, and provided direct confirmation of the high levels of X-ray emission from galaxies at these early times in the Universe (Lehmer et al. 2016). The new CDF-S 6 Ms data show that, at least to $z \approx 2.5$, the $2-10 \mathrm{keV}$ emission, originating primarily from XRB populations, evolves as $L_{2-10 k e V}(L M X B) / M^{*} \propto(1+z)^{2-3}$ and $L_{2-10 k e V}(H M X B) / S F R \propto(1+z)$. This evolution is consistent with basic predictions from XRB population synthesis models and the evolution is driven primarily by stellar aging and metallicity evolution of the Universe. XRB populations dominate in both the $0.5-2 \mathrm{keV}$ and $2-10 \mathrm{keV}$ bands; 


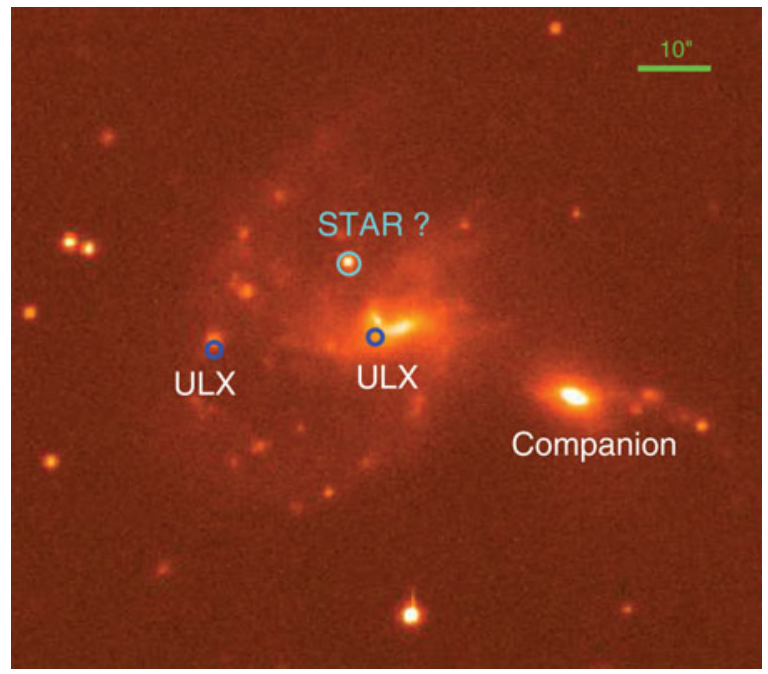

Figure 4. One example of the ring galaxies observed: AM0642-801. The image is the $\mathrm{H} \alpha$ net image from ESO-NTT in the HALR 709 filter. Labels indicate the companion galaxy, a possible foreground star (in cyan) and the two ULXs (in blue) detected in the Chandra data (A. Wolter et al., 2016, in prep). The total area is about $1.5 \times 1.5$ arcmin.

however, hot gas is inferred to provide significant contributions to the $0.5-2 \mathrm{keV}$ emissivity over the majority of cosmic history (Lehmer et al. 2016).

These results on the evolution of X-ray emission from galaxies provide strong observational support for the theoretically predicted significant role of XRBs in early heating of the primordial IGM (e.g., Fragos et al. 2013).

Vigorous starbursts: the ring galaxies (Wolter).

Intermediate-Mass black holes (IMBHs), i.e. objects with masses between $M_{\mathrm{BH}} \approx$ $10^{2-5} \mathrm{M}_{\odot}$, may be crucial for the initial seeding and growth of nuclear supermassive BHs in galaxies (Volonteri 2010), as sources of pre-heating of the IGM at early times, as discussed in the previous section (Fragos et al. 2013a, Mesinger et al. 2013), and of fluctuation in the Near IR Cosmic Background (Yue et al. 2013). Formation scenarios of IMBHs suggest that they can be born from primordial Population III stars (e.g., Madau \& Rees 2001), very dense stellar super-clusters through dynamical collapse of the central massive object (e.g. Portegies-Zwart et al. 2004), or repeated mergers (Miller \& Hamilton 2002). Identifying these objects is of crucial importance not only for assessing these scenarios, but also for establishing that IMBHs exist at all.

Among the best candidates for IMBHs are the brightest ULX sources (e.g. Colbert \& Mushotzky 1999) which are often observed as the most luminous point sources in galaxies. ULX sources, or ULXs, are extragalactic, point-like, off-nuclear X-ray sources characterized by X-ray luminosities, assumed to be isotropic, greater than $10^{39} \mathrm{erg} \mathrm{s}^{-1}$ (see Fabbiano et al. 2002 or Feng \& Soria 2011 for a review). Because of the high luminosities, peculiar accretion regimes, if the compact objects in question are stellar-mass black holes, are suggested. If instead, these ULXs had the higher masses of IMBHs, the need to invoke peculiar accretion regimes would be removed. Recent results support the notion that the majority of ULXs are in binary systems rather than at centers of galaxies and are likely accreting mass from a donor star (e.g., Swartz et al. 2004; Walton et al. 2011). 


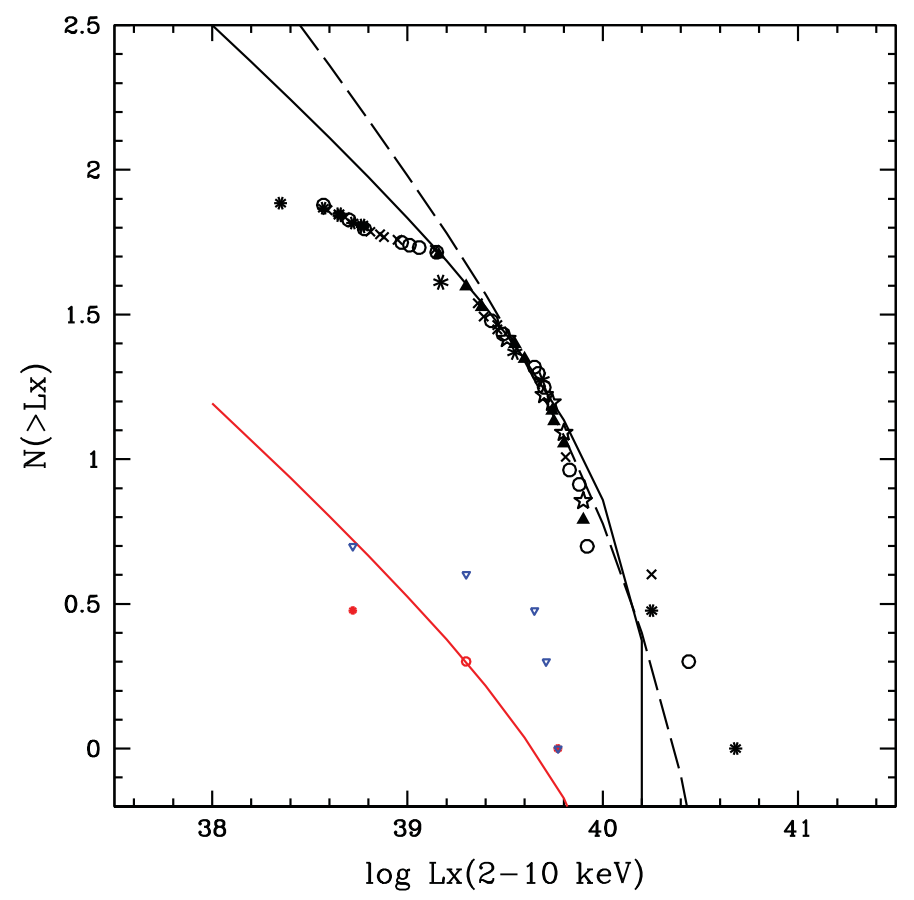

Figure 5. The total X-ray luminosity function (XLF) derived from the 6 observed ring galaxies (see text). The black solid line is the model of Grimm et al. (2003), normalized to SFR $=61$ $\mathrm{M}_{\odot} \mathrm{yr}^{-1}$, which is the sum of the SFR for the 6 "pointed" galaxies. The dashed line is the XLF from Swartz et al. (2011). In this plot, we consider only the sources associated to the rings. There are 50 sources in total, and 15 sources above $L_{X}>5 \times 10^{39} \mathrm{erg} \mathrm{s}^{-1}$. Different symbols correspond to the different galaxies. Four sources are about the cut-off limit and have $\mathrm{L}_{X}(2-10$ $\mathrm{keV})>10^{40} \mathrm{erg} / \mathrm{s}$. The red(blue) points are secure(all) sources from the "statistical" sample. The red solid line is the Grimm et al. (2003) model rescaled to SFR $=3 \mathrm{M}_{\odot} \mathrm{yr}^{-1}$.

If indeed ULXs host IMBHs, then an excellent environment in which to find them would be that of ring galaxies, a unique laboratory in which to study peculiar phases of galaxy evolution, including head-on collisions with a massive galaxy. The gravitational perturbation drives a density wave through the galaxy and its outskirts, involving also "pristine" (low metallicity) gas in the formation of new stars, generating an expanding ring of gas and stars (e.g. Lynds \& Toomre 1976). The simple geometry of ring galaxies allows us to reconstruct the main features of the interaction. Also, since the star formation in ring galaxies takes place only in the ring: the total surface area is smaller than in a "normal" galaxy, and therefore the association of X-ray sources with the galaxy itself is more likely than for a random place in the galaxy area. Furthermore, the high star formation rates $\left(\mathrm{SFR} \approx 0.1-20 \mathrm{M}_{\odot} \mathrm{yr}^{-1}\right.$ ) in ring galaxies testify to the fact that the density wave associated with the propagating ring indeed triggers star formation.

Most of the host galaxies that contain a large number of ULXs have been selected because they are bright and famous, e.g., the Cartwheel. The only four ring galaxies for which Chandra data have been published are the Cartwheel (Gao et al. 2003; Wolter \& Trinchieri 2004; Wolter, Trinchieri \& Colpi 2006; Crivellari et al. 2009; Pizzolato et al. 2010), Arp 284 (Smith et al. 2005), Arp 147 (Rappaport et al. 2010) and NGC 922 (Prestwich et al. 2012). We have analyzed archival data for two other collisional ring galaxy, AM 0644-741 and Arp 148 (see A. Wolter et al., 2016, in prep for details). 
Although a few hundred ULXs and candidates are now known, there has never been a specific survey tailored to find these sources. The collection of ULXs in various catalogs is based on detections without assessment of non-detections. As a first step towards creating a statistically significant sample of ULXs, we have also started a small but focused project to observe a sample of ring galaxies. We have selected all the peculiar galaxies labelled as "collisional rings" with a spectroscopic redshift $z<0.02$ from the Arp \& Madore (1987) 'Catalogue of southern peculiar galaxies and associations'. This selection produces a sample of 12 galaxies which we have observed with Chandra and $X M M-N e w t o n$. One of the galaxies is shown in Figure 4. Details of the analysis and results are described in A. Wolter et al., 2016, in prep. This pilot sample can also be used to plan deeper and wider surveys of IMBHs.

We have constructed the X-ray Luminosity Function (XLF) first from the "targeted" galaxies and then from the "statistical" sample that were chosen to be representative of the class (see details in A. Wolter et al. 2016, in prep.). The number of sources in the ULX regime is 16 in the Cartwheel, 9 in Arp 284 and Arp 147, 7 in NGC 922, at least 5 in AM 0644-741, and 3 in Arp 148 (assuming $\mathrm{H}_{0}=73 \mathrm{~km} \mathrm{~s}^{-1} \mathrm{Mpc}^{-1}$ and considering only sources along the ring). We correct for detection confusion following Kim et al. (2003). The flattening at the low luminosity end is due to the different detection limits in the various fields. We compare the resulting XLF to the models of Grimm et al. (2003) rescaled for the total SFR of the six galaxies and to the XLF of Swartz et al. (2011) for local galaxies (see Figure 5).

The number of sources detected in the "statistical" sample is small, five at most including two that might not be related to the rings, and does not allow us to reach firm conclusions. They are plotted in Figure 5 as blue (the five) or red (the secure three) points and are consistent with a total $\mathrm{SFR} \sim 5 \mathrm{M}_{\odot} \mathrm{yr}^{-1}$.

In the "targeted" sample, however, there is a definite excess of high $\mathrm{L}_{X}$ sources. If the low luminosity part of the ULX sample can be described as the tail of the HMXBs, these are definitely outliers. Are they really IMBHs? One possibility is again that they represent the extremes of the evolution of massive stars, reaching out to $120 \mathrm{M}_{\odot}$ for a metallicity of $\mathrm{Z}=0.01 \mathrm{Z}_{\odot}$ (see Spera et al. 2015). To investigate this further, we should enlarge the sample of "statistical" ring galaxies, by either observing more galaxies (including a Northern sample), or going deeper in flux.

\section{The $L_{\mathrm{X}, \mathrm{GAS}} T_{\mathrm{GAS}}$ Scaling Relation of Elliptical Galaxies (Kim)}

The X-ray scaling relations of early-type galaxies (ETGs) provide a powerful means to investigate the origin and evolution of the hot gas in these galaxies and its response to the forces that shape galaxy evolution, gravity and feedback - both stellar and AGN (e.g., Fabbiano 1989; Mathews \& Brighenti 2003; Boroson, Kim \& Fabbiano 2011; Kim \& Fabbiano 2013; Civano et al. 2014; Choi et al. 2014; Negri et al.2014). Based on decades of X-ray observations, and aided by the high resolution Chandra data that have allowed direct decomposition (spatial and spectral) of the emission components of nearby ETGs, two principal scaling relations have emerged: the $L_{\mathrm{X}, \mathrm{GAS}}-L_{\mathrm{K}}^{3-4}$ relation that links the X-ray emissivity of the hot gas with the total stellar mass of the galaxy, and the $L_{\mathrm{X}, \mathrm{GAS}}-T_{\mathrm{GAS}}^{4.5}$ relation, which describes the response of the hot gas to the many agents that drive its evolution. These scaling relations suggest that the hot gas is virialized, at least in X-ray luminous elliptical (E) galaxies (Kim \& Fabbiano 2013).

The scaling relations depend on the structural properties of the E galaxies (Kim \& Fabbiano 2015). Using the Atlas 3D sample, which has well characterized stellar kinematics and spatial distributions (Cappellari et al. 2011), we find that of the two scaling relations, the $L_{\mathrm{X}, \mathrm{GAS}}-T_{\mathrm{GAS}}^{4.5}$ is the tighter one. Moreover, this relation shows even less 


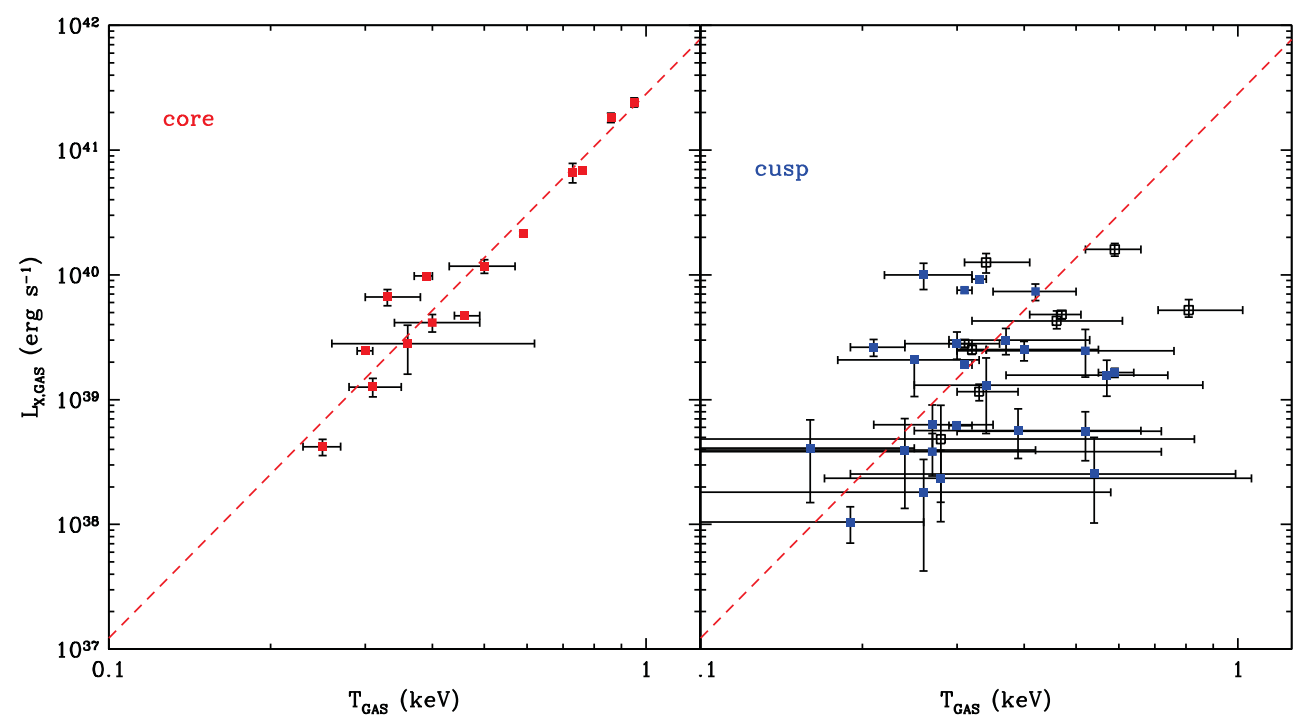

Figure 6. (a) $L_{\mathrm{X}, \mathrm{GAS}}$ plotted against $T_{\mathrm{GAS}}$ (a) for core and (b) coreless early type galaxies selected from the Atlas 3D sample. The core (cusp) galaxies are marked by red (blue) squares and those unidentified by black squares. The dashed line is the best fit relation $\left(L_{\mathrm{X}, \mathrm{GAS}}-T_{G A S}^{4.5}\right)$ among core galaxies.

dispersion, when only core Es are considered (with a rms deviation of 0.2 dex). Fig. 6 shows the $L_{\mathrm{X}, \mathrm{GAS}}-T_{\mathrm{GAS}}$ distribution for both core Es and for coreless Es (which include rotating galaxies). The $L_{\mathrm{X}, \mathrm{GAS}}-T_{\mathrm{GAS}}$ correlation of core Es appears to be a scaleddown version of similar relations found in $\mathrm{cD}$ galaxies and groups/clusters of galaxies (e.g., Pratt et al. 2009; O'Sullivan et al. 2003). We can qualitatively understand this correlation because larger galaxies both retain larger amounts of hot ISM and add more energy to the ISM from stellar and AGN feedback (e.g., Pellegrini 2011). In coreless Es instead a correlation is not present. Interestingly, a similarly large scatter is also seen in a sample of spiral galaxies (Li \& Wang 2013). The lack of correlation in coreless, rotating, partially star-forming Es may be due to their flattened potential and the presence of cold gas and dust, which affects the hot gas energy budget.

In Fig. 7, we further restrict the core E sample by excluding three galaxies, which have any sign of recent star formation from the Atlas 3D multi-wavelength data. These genuine E galaxies are characterized by central surface brightness cores, and slow or absent stellar bulk rotation, are $\sigma$-supported Es with old stellar populations. The rms deviation is only 0.13 dex. The ability to retain hot gas (potential depth) and the balance between heating and cooling seem to be closely regulated in all these galaxies. The fact that a single scaling relation holds in the entire genuine E sample suggests that genuine Es, when selected carefully, are a homogeneous sample. For $L_{\mathrm{X}, \mathrm{GAS}}>10^{40} \mathrm{erg}$ $\mathrm{s}^{-1}$, this correlation compares extremely well with the predictions of high resolution 2D hydrodynamical simulations for fully $\sigma$-supported galaxies (the shaded area in Fig. 7; Negri et al. 2014). However, the correlation extends down to $L_{\mathrm{X}, \mathrm{GAS}} \sim 10^{38.5} \mathrm{erg} \mathrm{s}^{-1}$, while simulations predict a sudden drop in X-ray luminosity in cooler galaxies, which appears in contradiction with the correlation. In fact, the ISM of gas-poor Es is likely to be in the outflow/wind state, and then tends to be at a temperature $\mathrm{T}_{\mathrm{GAS}}$ larger than that extrapolated from the $L_{\mathrm{X}, \mathrm{GAS}}-T_{\mathrm{GAS}}$ relation valid for inflows (the typical flow phase of gas-rich Es; e.g., Pellegrini 2011, Negri et al. 2014). What we are observing is 


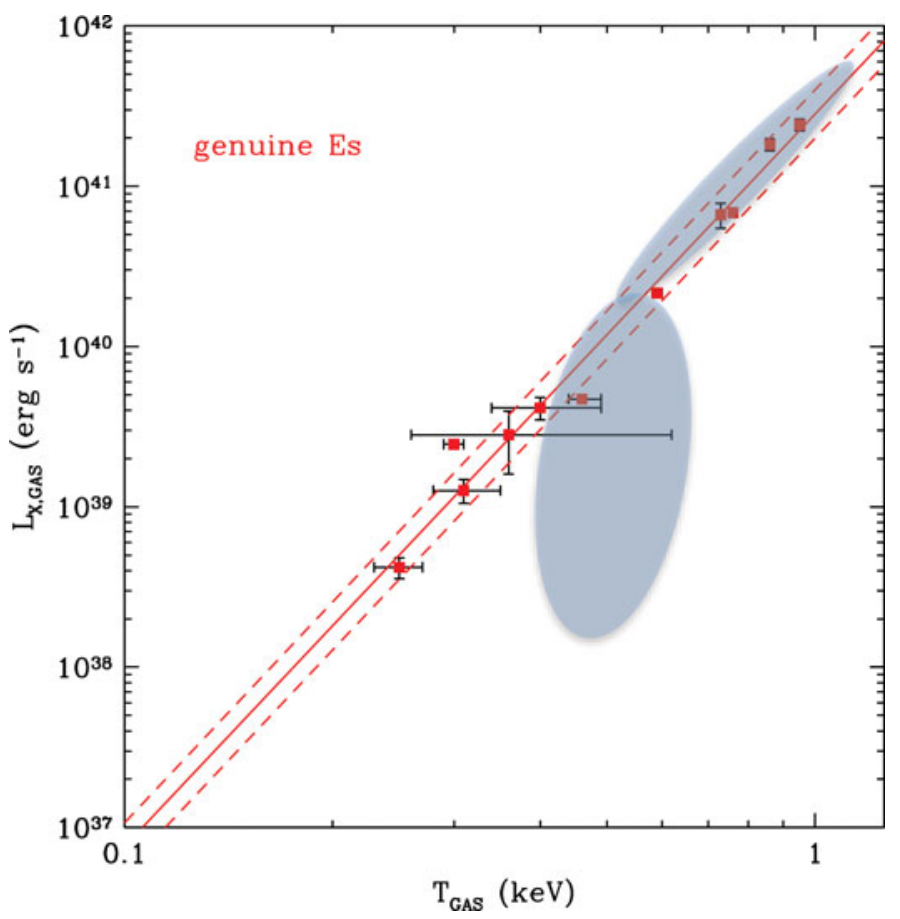

Figure 7. $L_{\mathrm{X}, \mathrm{GAS}}$ vs. $T_{\mathrm{GAS}}$ for a genuine E sample. The diagonal lines are the best fits (solid line) with rms deviations (dashed lines). The shaded region indicates the parameter space covered by $\sigma$-supported non-rotating Es taken from the numerical simulations of Negri et al. (2014, also including unpublished results).

instead the extension of the same correlation down to the lowest $\mathrm{L}_{\mathrm{X}}$. If this correlation at low $L_{\mathrm{X}, \mathrm{GAS}}$ is a real effect, it provides a new challenge to theoretical models. The sample in the existing study is small, particularly at lower luminosities, comprising only 6 galaxies with $L_{\mathrm{X}, \mathrm{GAS}}<10^{40}$ (Fig 7). It is important to observe more $\mathrm{X}$-ray faint genuine $\mathrm{E}$ galaxies to solidly establish the $L_{\mathrm{X}, \mathrm{GAS}^{-}} \mathrm{T}_{\mathrm{GAS}}$ relation and further provide strong constraints for theoretical modeling.

\section{Conclusion}

The topics described in these proceedings cover the most recent results on the most Xray luminous components of normal galaxies, including both stellar-mass and intermediatemass $\left(10^{2-5} M_{\odot}\right)$ compact objects in accreting binary systems and the emission from the hot phase of the ISM in the most massive elliptical galaxies. All of these components are critical to understanding the early heating of the IGM at Cosmic Dawn and there has been rapid progress the last several years with Chandra, XMM-Newton and NuSTAR.

One notable exciting observational prospect in the coming years is the pending launch of the Astro-H mission, which will enable spatially-resolved high spectral resolution studies of galaxies. This will be particularly helpful in ascertaining the nature of outflows in galaxies and the connection between galaxies and surrounding material (i.e., the IGM).

\section{Acknowledgements}

Ann Hornschemeier thanks collaborators Mihoko Yukita, Dan Wik, Bret Lehmer, Andrew Ptak, Tom Maccarone, Vallia Antoniou, Andreas Zezas and members of the $N u S$ - 
TAR team as well as Antara Basu-Zych, Bret Lehmer, Niel Brandt, Tassos Fragos, Bin Luo, Panayiotis Tzanavaris and Vicky Kalogera for the high- $z$ portion of this work. Ann Hornschemeier further acknowledges recent funding from Chandra GO grant GO213108Z, GO4-15130Z, and GO4-15806Z (P.I. Hornschemeier) and support through NASA contract no. NNG08FD60C, for the NuSTAR mission. Anna Wolter thanks collaborators Monica Colpi, Antonella Fruscione, Michela Mapelli, Fabio Pintore, Fabio Pizzolato, Emanuele Ripamonti, Ginevra Trinchieri, Luca Zampieri. Anna Wolter acknowledges funding from PRIN-2011-1 and ASI - INAF I/037/12/0. Dong-Woo Kim thanks collaborator Pepi Fabbiano and support from Chandra GO grant AR4-15005X and by 2014 Smithsonian Competitive Grant Program for Science.

\section{References}

Arp, H. \& Madore, B., 1987, Ap.\&S.S., 139, 196

Bachetti, M., et al. 2013, ApJ, 778, 163

Bachetti, M., et al. 2014, Nature, 514, 202

Basu-Zych, A. R., et al. 2013a, ApJ, 762, 45

Basu-Zych, A. R., et al. 2013b, ApJ, 774, 152

Basu-Zych, A. R., et al. 2016, ApJ, 818, 140

Boroson, B., Kim, D. W., \& Fabbiano, G. 2011, ApJ, 729, 12

Cappellari, M. et al. 2011, MNRAS, 413, 813

Civano, F. et al. 2014, ApJ, 790, 16

Colbert, E. J. M. \& Mushotzky, R. F., 1999, ApJ, 519, 89

Crivellari, E., Wolter, A., \& Trinchieri, G., 2009, A.EAAp., 501, 445

Fabbiano, G. 1989, ARA\&A, 27, 87

Fabbiano, G., 2006, ARA\&A, 44, 323

Feng, H. \& Soria, R., 2011, NewAR, 55, 166

Fragos, T., et al. 2013a, ApJ, 764, 41

Fragos, T., et al. 2013b, ApJL, 776, L31

Gao, Y., et al. 2003, ApJ, 596, 171

Gladstone, J. C., Roberts, T. P., \& Done, C. 2009, MNRAS, 397, 1836

Grimm, H.-J., Gilfanov, M., \& Sunyaev, R., 2003, MNRAS, 339, 793

Harrison, F. A., et al. 2013, ApJ, 770, 103

Hornschemeier, A. E., et al. 2002, ApJ, 568, 82

Hornschemeier, A. E., et al. 2003, AJ, 126, 575

Hornschemeier, A. E., et al. 2005, AJ, 129, 86

Kaaret, P. 2014, MNRAS, 440, L26

Kim, D.-W. \& Fabbiano, G. 2013, ApJ, 776, 116

Kim, D.-W. \& Fabbiano, G. 2015, ApJ, 812, 127

Lehmer, B. D., et al. 2005, AJ, 129, 1

Lehmer, B. D., et al. 2010, ApJ, 724, 559

Lehmer, B. D., et al. 2012, ApJ, 752, 46

Lehmer, B. D., et al. 2013, ApJ, 771, 134

Lehmer, B. D., et al. 2015, ApJ, 806, 126

Lehmer, B. D., et al. 2016, ApJ, in press (arXiv:1604.06461)

Li, J.-T. \& Wang, Q. D. 2013, MNRAS, 435, 3071

Linden, T., et al. 2010, ApJ, 725, 1984

Lynds, R. \& Toomre, A., 1976, ApJ, 209, 382

Maccarone, T. J., et al. 2016, MNRAS, 458, 3633

Madau, P. \& Rees, M. J., 2001, ApJ, 551, L27

Mathews, W. \& Brighenti, F. 2003, ARA\&A, 41, 191

Mesinger, A., Ferrara, A., \& Spiegel, D. S., 2013, MNRAS, 431, 621

Mesinger, A., Ewall-Wice, A., \& Hewitt, J. 2014, MNRAS, 439, 3262 
Miller, M. C. \& Hamilton, D. P., 2002, ApJ, 330, 232

Moran, E. C. et al. 1999, ApJ, 526, 649

Negri, A., et al. 2014, MNRAS, 445, 1351

O'Sullivan, E., et al. 2003, MNRAS, 340, 1375

Pacucci, F., Mesinger, A., Mineo, S., \& Ferrara, A. 2014, MNRAS, 443, 678

Pellegrini, S. 2011, ApJ, 738, 57

Pizzolato, F., Wolter, A., \& Trinchieri, G., 2010, MNRAS, 406, 1116

Portegies-Zwart S. F., et al., 2004, Nature, 428, 724

Pratt et al. 2009, A\&SA, 498, 361

Prestwich, A. H., et al. 2012, ApJ, 747, 150

Ptak, A. F., et al. 2015, ApJ, 104, 10

Rana, V., et al. 2015, ApJ, 799, 121

Rappaport, S., et al., 2010, ApJ, 721, 1348

Ryu, T. et al., 2016, MNRAS, 456, 223

Smith, B. J., et al., 2005, AJ, 130, 2117

Spera, M., Mapelli, M., \& Bressan, A., 2015, MNRAS, 451, 4086

Swartz, D. A., Ghosh, K. K., Tennant, A. F., \& Wu, K., 2004, ApJS, 154, 519

Swartz, D. A., Soria, R., Tennant, A. F., \& Yukita, M., 2011, ApJ, 741, 49

Volonteri, M., 2010, Nature, 466, 1049

Yue, B., et al., 2013, MNRAS, 433, 1556

Walton, D. J., Roberts, T. P., Mateos, S., \& Heard, V., 2011, MNRAS, 416, 1844

Walton, D. J., et al. 2013, ApJ, 779, 148

Wolter, A., Trinchieri, G., 2004, A\& $A p, 426,787$

Wolter, A., Trinchieri, G., \& Colpi, M., 2006, MNRAS, 373, 1627

Wik, D. R., et al. 2014, ApJ, 797, 79

Yukita, M., et al. 2016, ApJ, in press (arXiv:1604.07441) 